\title{
A study of antenna array based multipath propagation phenomenon in LAS-CDMA standard
}

\begin{abstract}
Throughout the history of wireless communications, spatially diversity's antenna array system has being important in improving the radio link between wireless users. Moreover, this system are proposed to reduce the multipath fading through its directivity narrowly radiation beam. The introduction of interference free window in delay domain from newly LASCDMA standard has received many attentions for furthering research on multipath delay spread. In this paper, we studied the effect of angular spread on maximum excess delay in multipath propagation environments. Then, we examined the application of antenna array in LAS-CDMA standard to overcome this multipath phenomenon. First of all, the multipath propagation literatures are reviewed, followed by the explanation of maximum angular spread in a simple discrete ring of scatterers model. Comparison analyses are made between conventional omnidirectional antenna and narrow beam antenna array. Finally, the analytical performances in term of power delay profile are presented.
\end{abstract}

Keyword: Multipath propagation; Antenna arrays; LAS-CDMA standard; Multipath delay spread 